\title{
A Research on the Effect of Worm Predation on Phosphorus Release from Actived Sludge
}

\author{
Huang Danping ${ }^{1}{ }^{\text {* }}$, Li Anran ${ }^{1}$, Li Lipin ${ }^{1}$, Zhang Jun ${ }^{1}$, Zuo Wei ${ }^{1}$, Tian Yu ${ }^{1}$, Liu Yingshi ${ }^{2}$ \\ ${ }^{1}$ School of Municipal and Environmental Engineering, Harbin Institute of Technology, Harbin, China \\ ${ }^{2}$ Guangzhou Municipal Engineering Design and Research Institute, Guangzhou, China
}

\section{Email address:}

1112720116@hit.edu.cn (Huang Danping)

${ }^{*}$ Corresponding author

\section{To cite this article:}

Huang Danping, Li Anran, Li Lipin, Zhang Jun, Zuo Wei, Tian Yu. A Research on the Effect of Worm Predation on Phosphorus Release from Actived Sludge. Science Discovery. Vol. 5, No. 3, 2017, pp. 217-222. doi: 10.11648/j.sd.20170503.20

Received: January 20, 2017; Accepted: March 17, 2017; Published: May 11, 2017

\begin{abstract}
The sludge reduction in the process of using worms, worms would destroy the sludge extracellular polymer institutions and bacteria, the soluble organic matter, nitrogen, phosphorus and other substances released into the water phase from sludge. By worms on sequencing batch experiments, this study is designed to investigate the sludge of phosphorus migration transformation process, reveals the effect of predation on the effect of phosphorus release in the sludge with worms. The research results that the sludge contains large amounts of phosphorus particles at the beginning, and after 24 hours, the phosphorus particles decomposed to the supernatant. At First, the upper supernatant phosphorus concentration reduced from $17.68 \mathrm{mg} / \mathrm{L}$ to $0.53 \mathrm{mg} / \mathrm{L}$, with the nitrate nitrogen concentration reduced significantly, speculated that the denitrifying phosphorus absorption occured in the worms bed at the beginning, as worms prey on phosphorus content in the supernatant and then gradually increased to $42.19 \mathrm{mg} / \mathrm{L}$; the phosphorus concentration of cell declined from $28.02 \mathrm{mg} / \mathrm{gMLSS}$ to 16.15 $\mathrm{mg} / \mathrm{gMLSS}$, which is $14.36 \%$ higher than the phosphorus concentration of the blank bed; the metal ions $\left(\mathrm{K}^{+}, \mathrm{Ca}^{2+}, \mathrm{Mg}^{2+}\right)$ concentration in the worms bed is a total of $66.89 \mathrm{mg} / \mathrm{L}$, about $48 \%$ higher than blank bed, speculated that the worm predation promoted the extracellular polymer metal ion release, thus promote the phosphorus release. With comprehensive analysis of the worms bed, resulted that the worms feeding effect significantly promoted the release of phosphorus in sewage sludge.
\end{abstract}

Keywords: Worms Prey, Denitrifying Dephosphatation, Phosphorus Particles, Release of Phosphorus

\section{蠕虫捕食对污泥中磷释放影响的研究}

黄丹平 ${ }^{1 *}$, 李安然 ${ }^{1}$, 李俐频 ${ }^{1}$, 张军 ${ }^{1}$, 左薇 ${ }^{1}$, 田禹 ${ }^{1}$, 刘颖诗 $^{2}$

${ }^{1}$ 市政环境工程学院, 哈尔滨工业大学, 哈尔滨, 中国

${ }^{2}$ 广州市市政工程设计研究院, 广州, 中国

邮箱

1112720116@hit. edu. cn（黄丹平）

摘要: 在利用蠕虫污泥减量的过程中, 蠕虫捕食作用会破坏污泥胞外聚合物及菌体机构, 使溶解性有机物、氮、磷等 物质大量从污泥中释放到水相中。本研究通过蠕虫捕食序批实验, 考察污泥中的磷的迁移转化过程, 揭示蠕虫捕食作 用对污泥中磷释放的影响。研究结果表明, 在捕食初期污泥中含有大量的聚磷颗粒, 而经过 $24 \mathrm{~h}$ 蠕虫捕食后污泥中的聚 磷颗粒分解转移到上清液中。蠕虫床中上清液磷浓度首先从 $17.68 \mathrm{mg} / \mathrm{L}$ 降低至 $0.53 \mathrm{mg} / \mathrm{L}$, 且硝态氮浓度显著降低, 推测 在捕食初期蠕虫床中发生了反硝化吸磷, 随着蠕虫捕食的进行上清液中磷含量又逐步增加到 $42.19 \mathrm{mg} / \mathrm{L}$; 蠕虫床细胞中 磷浓度从初始的 $28.02 \mathrm{mg} / \mathrm{gMLSS}$ 持续减少至 $16.15 \mathrm{mg} / \mathrm{gMLSS}$, 细胞中磷的释放量较空白蠕虫床提高了 $14.36 \%$; 蠕虫床上 
清液中金属离子 $\left(\mathrm{K}^{+} 、 \mathrm{Ca}^{2+} 、 \mathrm{Mg}^{2+}\right)$ 的浓度在捕食末期共达到 $66.89 \mathrm{mg} / \mathrm{L}$ ，比空白床高出约 $48 \%$ ，推测蠕虫捕食促进了胞 外聚合物中金属离子的释放, 进而促进了磷的释放。综合分析蠕虫床中磷的转移途径发现蠕虫的捕食作用显著促进了 污泥中磷的释放。

关键词: 蠕虫捕食, 反硝化吸磷, 聚磷颗粒, 磷释放

\section{1. 引言}

蠕虫捕食污泥减量技术通过延长污水处理工艺中的 食物链, 使物质和能量在食物链的传递过程中得到消耗, 实现污泥减量, 具有无需添加化学物质、无二次污染等优 势。在蠕虫捕食过程中, 污泥的胞外聚合物（EPS）及细 胞被破坏, 使得污泥中含有的溶解性有机物 ( $\mathrm{SCOD}$ ) 、氮、 磷等化合物大量释放到上清液中 [1]。课题组在前期研究 中建立了一种新型的蠕虫捕食生物反应器一蠕虫床, 实现 了污泥高效减量, 并通过控制蠕虫床缺氧/好氧交替条件, 在捕食过程中实现了同步硝化反硝化, 从而减轻了由于蠕 虫捕食所带来的COD和氮对污水处理负荷的影响 [2]。同时 实验发现蠕虫床上清液中磷的浓度大量增加, 推测与蠕虫 捕食破碎污泥细胞及EPS有关。

磷一般通过生物法去除, 分为好氧吸磷及厌氧释磷两 个过程 [3]。在好氧条件下, 聚磷菌分解细胞内的PHB提供 能量, 以氧为电子受体, 吸收上清液中的磷酸盐, 并进一 步合成聚磷颗粒; 厌氧条件下, 分解体内聚磷颗粒, 释放 磷酸盐至上清液中, 同时利用ATP吸收小分子有机物合成 有机颗粒PHB储存在细胞中。在缺氧条件下, 一部分聚磷 菌利用硝态氮作为电子受体, 分解PHB产生能量, 也可实 现磷的吸收过程 [4]。研究表明, 磷主要以聚磷和磷酸盐 的形式存在, 聚磷颗粒与磷酸盐大量存在于污泥的EPS中, 与金属离子 $\left(\mathrm{K}^{+} 、 \mathrm{Ca}^{2+} 、 \mathrm{Mg}^{2+} 、 \mathrm{Fe}^{3+} 、 \mathrm{Al}^{3+}\right)$ 络合, 作为骨架 支撑EPS, 以形成菌体细胞的保护层 [5]。EPS不仅起到保 护作用, 还维持着细胞内及上清液中磷的动态平衡。一般 认为, 蠕虫床对污泥中的磷表现为释放作用, 即通过捕食, 破坏细胞结构, 释放出体内的磷。但在蠕虫床的运行过程 中发现, 上清液中的磷浓度呈现先降低后增高的趋势, 这 可能与蠕虫床缺氧/好氧交替运行的条件有关, 即为反硝 化聚磷菌提供了缺氧环境, 利用蠕虫捕食释放的硝态氮作 为电子受体, 吸收上清液中的磷, 从而造成一段时间内的 磷浓度降低。基于此, 本文通过构建蠕虫床及空白床, 开 展序批实验, 进一步分析蠕虫对污泥中磷释放过程的影响。

\section{2. 材料与方法}

\section{1. 实验装置与污泥来源}

蠕虫床如图1所示, 反应器尺寸为 $25 \mathrm{~cm} \times 15 \mathrm{~cm} \times 16$ $\mathrm{cm}$ (长 $\times$ 宽 $\times$ 高), 有效容积为 $4 \mathrm{~L}$ 。反应器中有 4 个有机 玻璃材质的平板支撑, 上面放置多孔填料用于蠕虫附着, 蠕虫投量为 $100 \mathrm{~g}$ (湿重)。运行时, 长时间维持弱曝气提 供氧气供蠕虫生存, 间歇强曝气使蠕虫固定在填料上, 时
间为每小时强曝气3分钟。空白对照组除不加蠕虫外, 其 余条件与实验组相同。

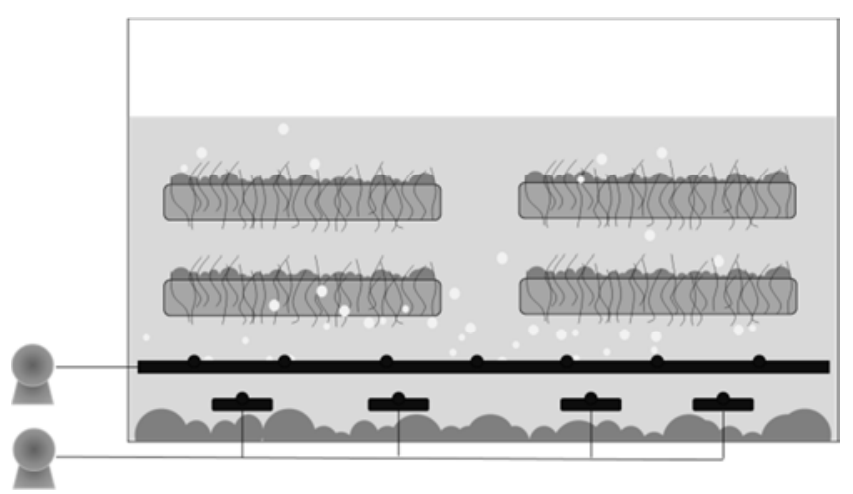

图1 蠕虫床及空白对照组示意图。

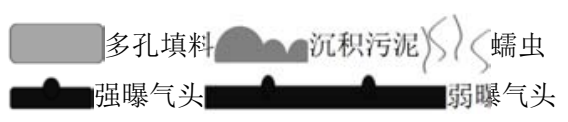

实验用活性污泥为好氧污泥, 来自哈尔滨文昌污水处 理厂二沉池, 其理化性质如表1所示。

表1 初始污泥的理化特性。

\begin{tabular}{ll}
\hline 项目 & 浓度 $(\mathrm{mg} / \mathrm{L})$ \\
\hline 悬浮固体浓度 (MLSS) & $4700-5000$ \\
挥发性悬浮固体浓度 (MLVSS) & $3300-3700$ \\
金属离子 $\left(\mathrm{K}^{+} 、 \mathrm{Ca}^{2+} 、 \mathrm{Mg}^{2+} 、 \mathrm{Fe}^{3+} 、 \mathrm{Al}^{3+}\right)$ & $\begin{array}{l}\mathrm{K}^{+}-20, \quad \mathrm{Ca}^{2+}-20, \quad \mathrm{Me}^{2+}-5, \\
\mathrm{Fe}^{3+}-0.5, \mathrm{Al}^{3+}-0.5\end{array}$ \\
\hline
\end{tabular}

\section{2. 实验运行方式}

实验采用双曝气系统强曝气 ( $\mathrm{D} 0=5 \sim 6 \mathrm{mg} / \mathrm{L})$, 弱曝气 ( $\mathrm{D} 0=0.5 \sim 1 \mathrm{mg} / \mathrm{L}), \mathrm{pH}$ 值 $7.0 \sim 7.5$, 温 度 $23 \sim 25^{\circ} \mathrm{C}$, $\mathrm{HRT}=3 \mathrm{~d}, \mathrm{SRT}=2 \mathrm{~d}$ 。每 $24 \mathrm{~h}$ 更换 $2 \mathrm{~L}$ 的污泥混合液。进泥和排泥 时采用强曝气, 使污泥混合均匀, 同时尽量避免蠕虫从填 料上脱落。在捕食阶段采用弱曝气与强曝气交替运行的方 式, 为蠕虫提供氧气。稳定运行后取初始样测定污泥浓度 及水质, 初始时刻为 0 时刻, 运行 $2 \mathrm{~h} 、 4 \mathrm{~h} 、 8 \mathrm{~h} 、 12 \mathrm{~h} 、 24 \mathrm{~h}$ 时各取样 $50 \mathrm{~mL}$, 进行相关分析。

\section{3. 分析测试方法}

\subsection{1. 常规指标检测方法}

实验中需检测指标为总磷（TP）、磷酸盐 $\left(\mathrm{PO}_{4}{ }^{3-}\right)$ 、 氨氮 $\left(\mathrm{NH}_{4}{ }^{+}-\mathrm{N}\right)$ 、硝态氮 $\left(\mathrm{NO}_{3}{ }^{-}-\mathrm{N}\right)$ 、MLSS、MLVSS、金属 离子 $\left(\mathrm{K}^{+} 、 \mathrm{Ca}^{2+} 、 \mathrm{Mg}^{2+} 、 \mathrm{Fe}^{3+} 、 \mathrm{Al}^{3+}\right)$ 。 
表2 常规检测指标及方法。

\begin{tabular}{|c|c|}
\hline 项目 & 检测方法 \\
\hline TP & 过二硫酸钾消解-钿锑抗显色分光光度法 \\
\hline $\mathrm{PO}_{4}^{3-}$ & 钿锑抗显色分光光度法 ～～～～ \\
\hline $\mathrm{NH}_{4}^{+}-\mathrm{N}$ & 纳氏试剂显色分光光度法 \\
\hline $\mathrm{NO}_{3}^{-}-\mathrm{N}$ & 氨基磺酸氨-摩香草酚显色分光光度法 \\
\hline MLSS & 称重法 \\
\hline MLVSS & 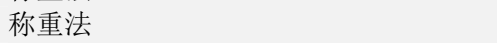 \\
\hline 金属离子 & 电感耦合等离子体发射光谱法 \\
\hline
\end{tabular}

\subsection{2. 其他指标检测方法}

上清液提取：取污泥混合液 $50 \mathrm{~mL}$, 在 $4000 \mathrm{rpm}$ 下离心 $5 \mathrm{~min}$, 上清液过 $0.45 \mu \mathrm{m}$ 滤膜, 滤液为上清液样品。

EPS提取 [6]: 采用酸提法提取EPS, 将提取过SMP的污 泥加入缓冲溶液补齐 $50 \mathrm{~mL}$, 再加入 $8 \%$ 硫酸 $50 \mathrm{~mL}$, 在 $300 \mathrm{rpm}$ 的条件下振荡提取 $1 \mathrm{~h}$ 后, 在 $4000 \mathrm{rpm}$ 下离心 $5 \mathrm{~min}$, 上清液 过0. $2 \mu \mathrm{m}$ 滤膜, 滤液为EPS样品。

细胞内物质提取 [7]: 采用超声破碎+热提法提取细胞 内物质, 取上述提取过EPS的污泥加入 $0.85 \% \mathrm{NaCl}$ 补齐 $50 \mathrm{~mL}$, 在 $20 \mathrm{KHz}$ 下超声破碎 $2 \mathrm{~min}$, 转移到 $80^{\circ} \mathrm{C}$ 的恒温水浴锅中加 热 $30 \mathrm{~min}$, 取上清液过 $0.2 \mu \mathrm{m}$ 滤膜, 滤液为细胞内物质样 品。

缓冲溶液配制: 准确称取 $\mathrm{Na}_{3} \mathrm{PO}_{4} 0.7600 \mathrm{~g}, \mathrm{NaH}_{2} \mathrm{PO}_{4}$ $0.6240 \mathrm{~g}, \mathrm{NaCl} \quad 0.5260 \mathrm{~g}, \mathrm{KCl} \quad 0.0745 \mathrm{~g}$, 加入去离子水中, 定容至 $1000 \mathrm{~mL}$ 。

PHB染色方法: 尼罗蓝染色法 $[8]$, 取 $1 \mathrm{~g} / \mathrm{L}$ 尼罗蓝染液 1 2滴滴在污泥涂片上, 染色 10-30s, 清水冲洗染色液, 自然风干后, 于荧光显微镜 $(460 \mathrm{~nm} \times 546 \mathrm{~nm})$ 下观察。

聚磷颗粒染色方法: 亚甲基蓝染色法, 取 $1 \mathrm{~g} / \mathrm{L}$ 亚甲基 蓝染液1 2滴滴在污泥涂片上, 染色 $10^{\sim} 30 \mathrm{~s}$, 清水冲洗风 干，于显微镜下观察。

\section{3. 结果与讨论}

蠕虫床及空白床稳定运行一周后, 由于减量效果不同, 蠕虫床及空白床污泥浓度及性质出现区别, 各取 $2 \mathrm{~L}$ 相同 性质的好氧污泥同时排入蠕虫床及空白床, 作为序批实验 的开始。

\section{1 . 染色结果分析}

将排入和排出蠕虫床的污泥制片染色，图 $2 \mathrm{a}$ 、图 $2 \mathrm{~b}$ 为PHB染色结果, PHB呈红色; 图2c、图 $2 \mathrm{~d}$ 为聚磷颗粒染色 结果, 其中蓝黑色为细胞物质, 粉紫色为聚磷颗粒。对比 发现, 排入蠕虫床的污泥中含有较多的聚磷颗粒和少量的 PHB, 经捕食后污泥中聚磷颗粒染色数量变少, 且色块变 小, PHB含量也有所降低。推测是排入蠕虫床的污泥为好 氧污泥, 经过好氧吸磷作用消耗PHB, 将游离的磷酸盐形 成聚磷颗粒大量储存在EPS及细胞中。排出蠕虫床的污泥 经过 $24 \mathrm{~h}$ 蠕虫捕食后, 加剧破坏了细胞及EPS的结构, 使 聚磷颗粒分解释放, 导致聚磷颗粒含量的降低; 同时, 由 于蠕虫床缺氧/好氧交替运行方式, 大部分时间为缺氧条 件, 使得反硝化聚磷菌分解利用PHB, 进一步造成PHB含量 的降低。

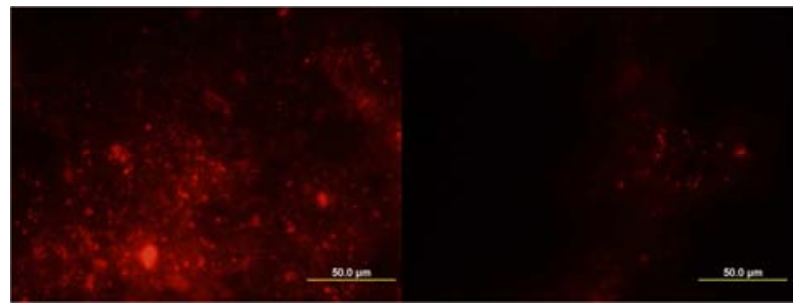

(a)

(b)

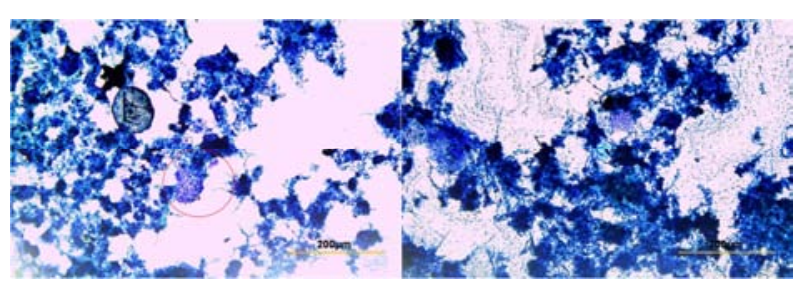

(c)

(d)

图2 排入及排出蠕虫床污泥中PHB及聚磷颗粒染色结果。

\section{2. 磷浓度变化分析}

序批实验开始后, 将运行过程中取得的样品进行上清 液、EPS及细胞中磷含量的提取与分析, 测定磷酸盐、总 磷浓度, 结果如下所示。

\subsection{1. 上清液磷浓度及氮浓度变化分析}

蠕虫床及空白床上清液磷浓度的变化如图3所示, 氨 氮及硝态氮浓度变化趋势如图4所示。在空白床中经过 $24 \mathrm{~h}$ 反应后, 上清液总磷浓度由 $3.32 \mathrm{mg} / \mathrm{L}$ 增加至 $13.27 \mathrm{mg} / \mathrm{L}$, 磷酸盐浓度变化趋势与总磷一致。在前 $4 \mathrm{~h}$ 内, 上清液总磷 浓度先降低至 $0.74 \mathrm{mg} / \mathrm{L}$, 与此同时硝态氮浓度从 8.65 $\mathrm{mg} / \mathrm{L}$ 逐渐降低, 推测污泥在缺氧环境中发生了反硝化吸磷 作用, $4 \mathrm{~h}$ 后上清液磷浓度逐渐升高, 是由于污泥衰减破损, 释放出细胞体内的磷。最终上清液总磷浓度比初始时提高 9. $95 \mathrm{mg} / \mathrm{L}$ 。

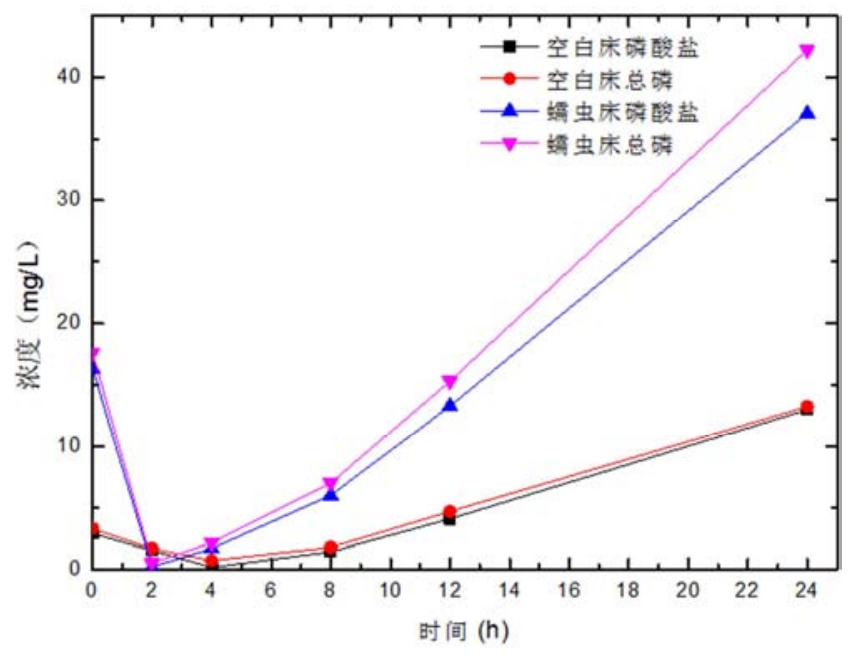

图3 上清液中磷浓度变化。 


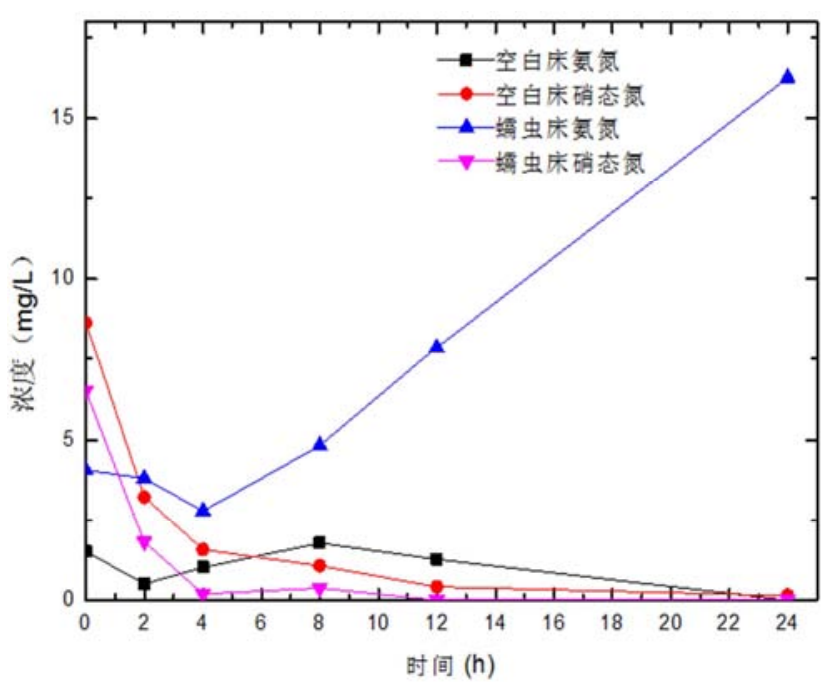

图4 上清液中氨氮、硝态氮浓度变化。

在蠕虫床缺氧条件下, 聚磷菌利用硝酸盐作为电子 受体, 分解体内的PHB提供能量, 吸收上清液中磷酸盐 到EPS中形成聚磷颗粒, 同时合成ATP [9], 造成上清液 中磷浓度在前 $2 \mathrm{~h}$ 内从 $17.68 \mathrm{mg} / \mathrm{L}$ 下降至 $0.53 \mathrm{mg} / \mathrm{L}$, 与 空白床对比看来, 蠕虫床在前 $2 \mathrm{~h}$ 内完成了对上清液中 总磷的吸收, 其吸收速率为 $8.58 \mathrm{mg} / \mathrm{L} \cdot \mathrm{h}$, 空白床吸收 速率约为 $0.65 \mathrm{mg} / \mathrm{L} \cdot \mathrm{h}$, 蠕虫床吸收速率约为空白床的 13 倍, 表明经蠕虫床驯化后的污泥可能有更强的吸磷能 力。随着反应进行, 硝态氮浓度由 $6.51 \mathrm{mg} / \mathrm{L}$ 持续降低, 氨氮浓度先由 $4.06 \mathrm{mg} / \mathrm{L}$ 降低至 $2.79 \mathrm{mg} / \mathrm{L}$, 表明在蠕虫 床中存在同步硝化反硝化作用。随着反应进行, 硝态氮 浓度趋于 0 , 反硝化吸磷作用速率减缓, 而蠕虫捕食仍 在破碎细胞及EPS, 释放出内部的氨氮及磷, 最终上清 液磷和氨氮浓度分别达到 $42.19 \mathrm{mg} / \mathrm{L} 、 16.26 \mathrm{mg} / \mathrm{L}$ 。蠕 虫床上清液增加量约为空白床增加量的 2.4 倍, 表明蠕 虫的捕食促进了污泥磷的释放。

综上, 在蠕虫床及空白床中发生反硝化作用的同时, 聚磷菌利用硝态氮为电子受体, 进行了反硝化吸磷作用对 磷进行吸收, 使得上清液磷浓度在短时间内降低。而当硝 态氮浓度降低至 0 时, 反硝化吸磷作用速率减缓, 使释放 出的磷大于吸收的磷, 最终表现为上清液磷浓度增加。蠕 虫床由于蠕虫的捕食作用, 细胞破坏的速率更大, 使上清 液中的磷含量比空白床更高。

\subsubsection{EPS磷浓度变化分析}

空白床及蠕虫床中污泥EPS磷含量的变化如图5所示。 空白床中EPS总磷浓度稳定在 $30 \mathrm{mg} / \mathrm{gMLSS}$ 左右, 磷酸盐浓 度稳定在 $24 \mathrm{mg} / \mathrm{gMLSS}$ 左右。与上清液中不同, EPS中总磷 及磷酸盐的差值较大, 表明在EPS中可能存在大量的聚磷 颗粒。

蠕虫床中EPS总磷与空白床相比具有不同变化趋势, 在前 $2 \mathrm{~h}$ 内, EPS中总磷及磷酸盐浓度增加, 其增加的原 因可能是由于聚磷菌的反硝化吸磷作用更强, 可吸收更 多上清液中的磷储存在EPS中。随着反应的进行, 蠕虫 的捕食使EPS及细胞结构破坏, 造成上清液浓度持续增 加, 而EPS的浓度在 $40^{\sim} 50 \mathrm{mg} / \mathrm{L}$ 范围内波动, 表明EPS在
磷的扩散过程中充当了一个中转站, 即联通细胞和上清 液, 用于磷的暂时储存和运输, 维持着磷浓度的动态平 衡。

经过 $24 \mathrm{~h} 反$ 应, 污泥由于蠕虫捕食（污泥衰减）的刺 激, 污泥大量分泌EPS, 与刚投入蠕虫床 (空白床) 时相 比, 污泥含量减少, 但单位污泥重量的EPS总含量增加, 未被破坏EPS加大了对上清液磷的吸收作用, 使得EPS中磷 含量增加, 但由于EPS含量也增加了, 所以单位EPS磷浓度 可能维持了动态平衡。同时, 蠕虫床EPS总磷浓度比空白 床高约 $11 \mathrm{mg} / \mathrm{gMLSS}$, 推测蠕虫大量破坏污泥结构, 污泥 分泌出更多的EPS, 吸收大量磷储存在EPS中, 使蠕虫床EPS 含磷量高于空白床。表明经过蠕虫床的捕食作用, 可能加 强了污泥的吸磷能力, 使得EPS中磷总量增加, 但单位EPS 磷浓度维持平衡。

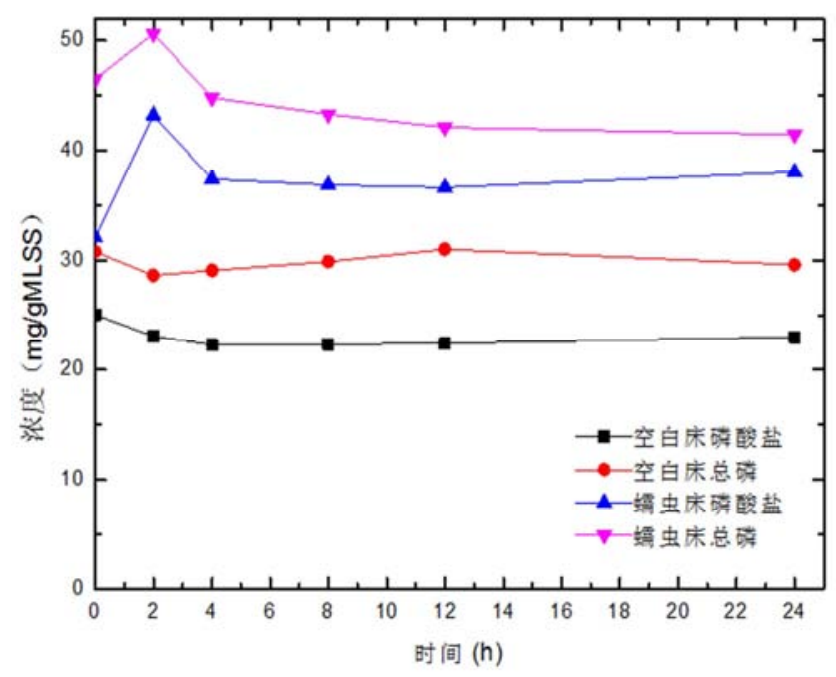

图5 EPS中磷浓度变化。

\subsection{3. 细胞中磷浓度变化分析}

空白床及蠕虫床污泥细胞中磷含量变化如图6所示。 在缺氧环境下, 当硝态氮存在时, 反硝化聚磷菌利用硝 态氮作为电子受体, 吸收上清液中磷, 形成聚磷颗粒储 存在EPS及细胞中; 当硝态氮消耗殆尽时, 细菌则利用 上清液中的 sCOD, 进行缺氧释磷作用 [10]。在反应前期, 虽然发生了反硝化吸磷, 但细胞中的磷含量并没有增加, 而是逐步降低。推测其可能的原因是污泥通过吸磷作用 将磷储存在EPS中, 未转移到细胞内部, 而随着反应进 行, 污泥释磷作用占主导作用, 导致细胞内磷浓度的逐 渐降低。其中空白床中细胞内总磷含量由 $14.55 \mathrm{mg} / \mathrm{gMLSS}$ 降低到 $10.41 \mathrm{mg} / \mathrm{gMLSS}$, 蠕虫床中由 $28.02 \mathrm{mg} / \mathrm{gMLSS}$ 降低至 $16.15 \mathrm{mg} / \mathrm{gMLSS}$, 两个反应器中 磷释放量的差异可能与上清液 SCOD浓度有关。在蠕虫床 中由于捕食作用使得 SCOD浓度较空白蠕虫床高, 促进了 蠕虫床中污泥的释磷能力, 使磷的释放量较空白蠕虫床 提高了 $14.36 \%$ 。 


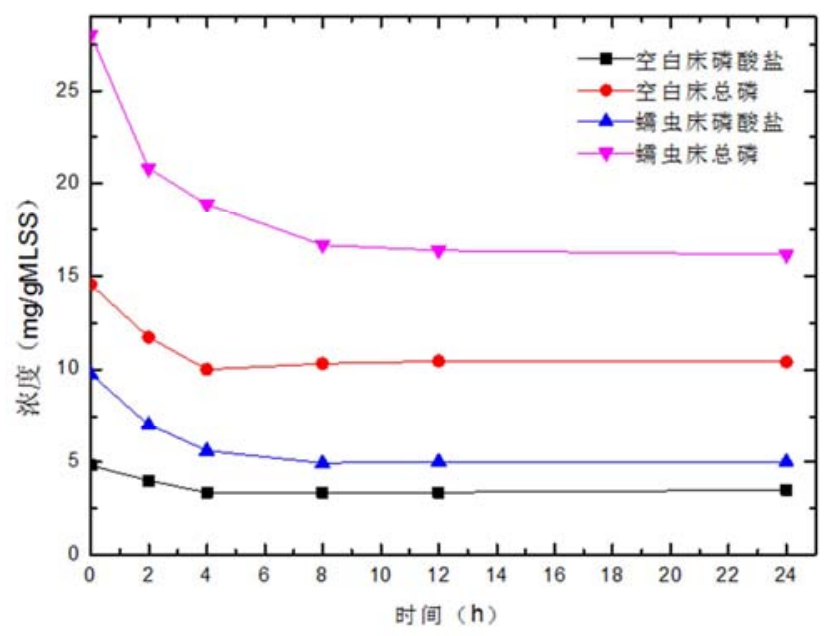

图6 细胞中磷浓度变化。

\section{3. 金属离子释放分析}

污泥在好氧状态时, 分解PHB产能, 吸收上清液中金 属离子 $\mathrm{K}^{+} 、 \mathrm{Ca}^{2+} 、 \mathrm{Mg}^{2+}$ 等进入 $E P S$ 或细胞中，与游离的磷酸 根结合, 形成聚磷配体, 储存在细胞中; 而在厌氧状态下, 聚磷配体分解为磷酸根和金属离子, 扩散到上清液中 [11]。 这一过程与污泥吸磷释磷过程吻合, 因此, 上清液中金属 离子的浓度的变化可以侧面显示细胞的吸磷或释磷过程。 $\mathrm{Fe}^{3+} 、 \mathrm{Al}^{3+}$ 与磷酸根结合, 降低上清液中磷酸根离子浓度, 形成磷酸铁、磷酸铝等难溶物 [12]。将两组实验所取样品 上清液进行 ICP-OES测定 $\mathrm{K}^{+} 、 \mathrm{Ca}^{2+} 、 \mathrm{Mg}^{2+} 、 \mathrm{Fe}^{3+} 、 \mathrm{Al}^{3+}$ 各离子 的含量, 测定结果如图7、图8所示。

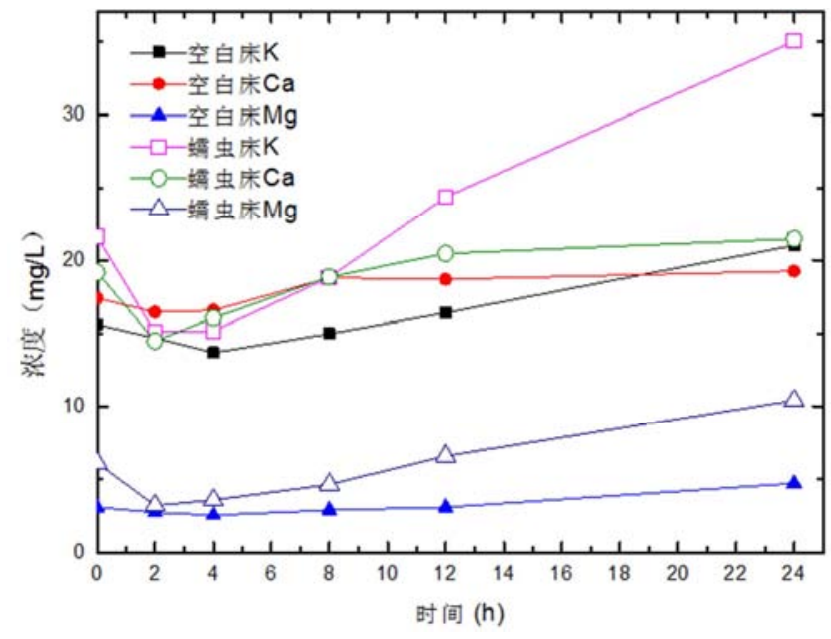

图7 上清液中金属离子 $\left(\mathrm{K}^{+} 、 \mathrm{Ca}^{2+} 、 \mathrm{Mg}^{2+}\right)$ 浓度变化。

图7中反映了 $\mathrm{K}^{+} 、 \mathrm{Ca}^{2+} 、 \mathrm{Mg}^{2+}$ 离子随取样时间的变化。 空白床中, 前 $4 \mathrm{~h}$ 内, 离子浓度略有降低, 而后 $\mathrm{K}^{+} 、 \mathrm{Ca}^{2+} 、$ $\mathrm{Mg}^{2+}$ 的浓度分别缓慢升高至 $21.02 \mathrm{mg} / \mathrm{L} 、 19.26 \mathrm{mg} / \mathrm{L}$ 和 $4.69 \mathrm{mg} / \mathrm{L}$ 。蠕虫床从开始的 0 时刻到 $2 \mathrm{~h}$ 期间, 离子浓度均 降低, 表示在序批反应的前 $2 \mathrm{~h}$ 内, 污泥在缺氧条件下吸收 摄入了上清液中 $\mathrm{K}^{+} 、 \mathrm{Ca}^{2+} 、 \mathrm{Mg}^{2+}$ 离子, 进入EPS或细胞中, 结合同时被吸入的磷酸根, 形成配体, 造成EPS中磷酸盐 浓度的增加。在蠕虫逐渐捕食, 污泥絮体破碎过程中, 上
清液 $\mathrm{K}^{+} 、 \mathrm{Ca}^{2+} 、 \mathrm{Mg}^{2+}$ 离子浓度逐渐升高, $24 \mathrm{~h}$ 周期结束后, 上清液中 $\mathrm{K}^{+} 、 \mathrm{Ca}^{2+} 、 \mathrm{Mg}^{2+}$ 浓度分别达到 $34.99 \mathrm{mg} / \mathrm{L} 、 21.49 \mathrm{mg} / \mathrm{L}$ 和 $10.41 \mathrm{mg} / \mathrm{L}$, 均大于初始浓度值与空白床浓度值，金属 离子总量比空白床高出约 $48 \%$ 。这可能是因为蠕虫捕食加 速释放出磷酸盐与金属离子的配体, 导致蠕虫床上清液金 属离子浓度增量均比空白床更大, 其中 $K^{\dagger}$ 浓度增量最大, 约为空白床的 2.6 倍。

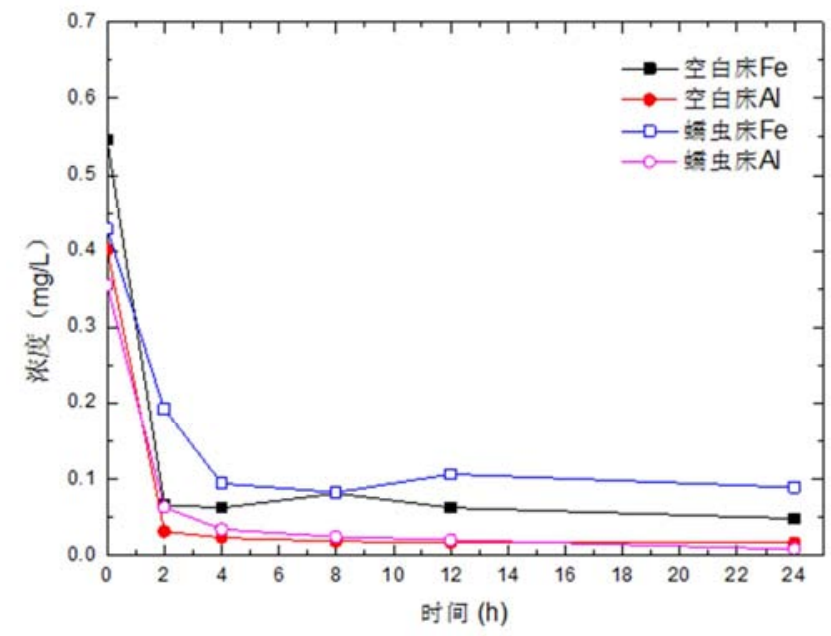

图8 上清液中金属离子 $\left(\mathrm{Fe}^{3+} 、 \mathrm{Al}^{3+}\right)$ 浓度变化。

如图8所示, 上清液中 $\mathrm{Fe}^{3+} 、 \mathrm{Al}^{3+}$ 含量变化呈现出不同 的趋势, 这两种离子的初始浓度值均小于 $0.6 \mathrm{mg} / \mathrm{L}$, 在整 个反应过程中, 浓度均呈降低趋势, 这是因为 $\mathrm{Fe}^{3+} 、 \mathrm{Al}^{3+}$ 易和上清液中磷酸盐形成难溶物, 沉淀到污泥中, 对上清 液中磷酸盐有一定的去除作用。但因为细胞中 $\mathrm{Fe}^{3+} 、 \mathrm{Al}^{3+}$ 含量较低, 通过沉淀作用去除的磷的量相比上清液的磷含 量十分微弱。

\section{4. 蠕虫床对污泥中磷转移的分析}

经过 $24 \mathrm{~h}$ 的序批实验过程, 空白床上清液、EPS及细胞 中磷含量分别是 $13.27 \mathrm{mg} / \mathrm{L} 、 29.58 \mathrm{mg} / \mathrm{gMLSS} 、 10.41$ $\mathrm{mg} / \mathrm{gMLSS}$; 蠕虫床为 $42.19 \mathrm{mg} / \mathrm{L} 、 41.46 \mathrm{mg} / \mathrm{gMLSS} 、 16.15$ $\mathrm{mg} / \mathrm{gMLSS}$ 。蠕虫床对应值均比空白床高, 其原因是由于蠕 虫捕食比单纯的污泥衰减对污泥破坏性更大, 造成污泥细 胞及EPS破损更多, 释放出更多细胞中的磷到上清液中; 与此同时，未被破坏的污泥分泌出更多的EPS，通过反硝 化作用吸磷使得EPS中磷含量保持稳定。导致到最终 $24 \mathrm{~h}$ 后, 污泥浓度降低、EPS增加、细胞含量减少, 上清液磷 浓度增加, EPS磷浓度平衡波动, 细胞内磷浓度降低。

综合磷浓度与金属离子浓度变化来看, 蠕虫床中污泥 磷的释放经历了如图 9 所示过程, 即在缺氧状态下时, 聚 磷菌分解体内的PHB, 以硝态氮作为电子受体, 吸收上清 液中的磷, 合成聚磷颗粒储存在EPS中, 完成缺氧反硝化 吸磷作用。再通过蠕虫的捕食破坏EPS及菌体细胞结构, 使得原本储存在EPS及细胞体内的聚磷颗粒、磷酸盐及与 聚磷结合的金属离子 $\left(\mathrm{K}^{+} 、 \mathrm{Ca}^{2+} 、 \mathrm{Mg}^{2+}\right)$ 释放到上清液中 [13]。 与刚投入蠕虫床的污泥相比, 经过 $24 \mathrm{~h}$ 蝡虫捕食, 污泥结 构及EPS遭到破坏, 刺激了菌体的自我保护机制, 分泌更 
多的EPS加大吸磷储磷能力 [14]; 同时, 上清液中的 $\mathrm{K}^{+}$、 $\mathrm{Ca}^{2+} 、 \mathrm{Mg}^{2+}$ 离子与磷酸盐结合, 可再次被吸收至EPS及细胞 中。

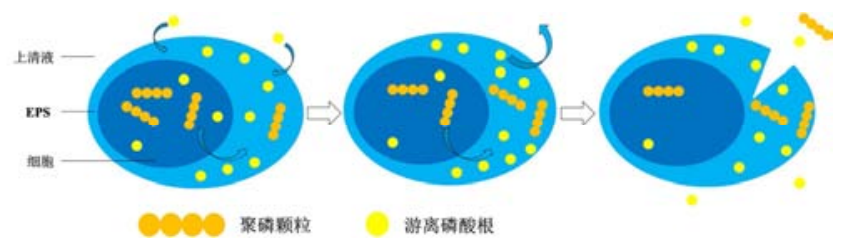

图9 蠕虫床中污泥磷转移示意图。

\section{4. 结论}

空白床及蠕虫床中为缺氧/好氧交替环境, 为反硝化 聚磷菌提供了反应条件, 好氧污泥在空白床及蠕虫床中发 生了磷的释放。在空白床中, 排入污泥上清液磷浓度由 $3.32 \mathrm{mg} / \mathrm{L}$ 增加至 $13.27 \mathrm{mg} / \mathrm{L}$; EPS含磷量稳定在 $30 \mathrm{mg} / \mathrm{gMLSS}$ 左右; 细胞内含磷量由 $14.55 \mathrm{mg} / \mathrm{gMLSS}$ 减少到 $10.41 \mathrm{mg} / \mathrm{gMLSS}$ 。在蠕虫床中, 上清液磷浓度 $17.68 \mathrm{mg} / \mathrm{L}$ 上升到 $42.19 \mathrm{mg} / \mathrm{L}$; EPS 含磷量维持在 $40 \sim 50 \mathrm{mg} / \mathrm{gMLSS}$ 范围 内; 细胞内含磷量由 $28.02 \mathrm{mg} / \mathrm{gMLSS}$ 减少到 $16.15 \mathrm{mg} / \mathrm{gMLSS}$ 。 造成空白床及蠕虫床变化差异的原因如下。

a）蠕虫床及空白床上清液磷浓度均呈现先降低后升 高趋势。推测由于反硝化吸磷作用造成上清液短时间内降 低, 同时由于污泥衰减或蠕虫捕食导致上清液浓度增加。 蠕虫床反硝化吸磷速率约为空白床的 13 倍, 表明经蠕虫床 驯化后的污泥可能有更强的吸磷能力。

b）经过蠕虫捕食及污泥衰减, 污泥结构及EPS遭到破 坏, 未被破坏的污泥分泌出更多EPS, 使EPS含量增加, 吸 磷作用增强。在经缺氧吸磷到缺氧释磷过程中, EPS中总 磷含量保持动态平衡。

c）硝态氮的浓度影响了污泥吸磷释磷的作用, 在反 应前期反应器中硝态氮浓度较高, 聚磷菌发生缺氧吸磷作 用, 将上清液中磷吸收储存在EPS中; 随着硝态氮浓度的 降低, 反硝化吸磷作用逐渐降低, 污泥逐步表现出庆氧状 态下的释磷作用, 使污泥细胞中磷浓度持续降低。空白床 中, 污泥释放出细胞内部约 $28 \%$ 的磷; 蠕虫床中由于较高 的上清液 $\mathrm{SCOD}$ 的浓度促进了污泥的的厌氧释磷能力, 释放 出细胞内部约 $42 \%$ 的磷。

d）由于蠕虫的捕食作用，促进了污泥细胞中金属离 子的释放过程, 金属离子与磷的配位作用减弱, 与金属离 子结合的磷被释放到上清液中, 进一步导致蠕虫床中上清 液磷增加量大于空白床。

\section{致谢}

本研究受到广东省广州市市政工程设计研究院城市 环境治理院士工作站（2013B090400006）的支持。

\section{参考文献}

[1] 邓胜平. CAST+斜板蠕虫附着床污泥减量工艺实验研究 [D]. 重庆: 重庆大学, 2014。

[2] Yu Tian, Yaobin Lu. Simultaneous nitrification and denitrification process in a new Tubificidae-reactor for minimizing nutrient release during sludge reduction[J]. Water Research, 2010, 44: 6031-6040.

[3] Ekama, George A. Recent developments in biological nutrient removal [J]. WATER SA, 2015, 41(4) : 515-524.

[4] Qin Lu, Haiyan Wu, Haoyan Li, etc. Enhanced biological nutrient removal in modified carbon source divisionanaerobic anoxic oxic process with return activatedsludge pre-concentration $[J]$. Chinese Journal of Chemical Engineering, 2015, 23(6) : 1027-1034.

[5] Wenwei Li, Hailing Zhang, Guoping Sheng, etc. Roles of extracellular polymeric substances in enhanced biological phosphorus removal process[J]. Water Research, 2015, 86: 85-95.

[6] 郭超, 刘怀英, 王琪, 等. 除磷颗粒污泥中磷的形态及其 含量分布 $[J]$ 。安全与环境工程, 2015, 22 (2) : 44-47。

[7] 李继宏, 单士亮, 李亮, 等. 膜生物反应器中EPS的提取方 法 [J]. 环境工程, 2013, 31 (3) : 10-14。

[8] 葛艳辉, 赵林, 周艳. 聚磷菌胞内聚合物的染色条件优化 及染色方法比较 $[J] .2014,27(2): 1-4$ 。

[9] 刘丹丹, 李慧, 陈文清. 反硝化聚磷菌脱氮除磷机理研究 [J]四川化工，2015，18(2):1-4。

[10] 叶丽红, 李冬, 张杰, 等. 亚硝化一反硝化除磷技术研究进 展 $[J]$. 北京工业大学学报, 2016, 42 (4) :586-589。

[11] 唐悦恒, 方闻, 罗荣, 等. SBR/OSA工艺的污泥减量化运行 机理研究 $[J]$.中国给水排水, 2011, 27 (5): 104-108。

[12] Jong-Oh Kim, Jinwook Chung. Implementing Chemical Precipitation as a Pretreatment for Phosphorus Removal in Membrane Bioreactor-Based Municipal Wastewater Treatment Plants[J]. Environmental Engineering, 2014, 18(4): 956-963.

[13] T. L. G. Hendrickx, H. Temmink, H. J. H Elissen, etc. Aquatic worms eat sludge: Mass balance and processing of worm faeces[J]. Journal of Hazardous Materials, 2010, $177(1-3)$ : 633-638.

[14] Yayi Wang, Jian Qin, Shuai Zhou, etc. Identification of the function of extracellular polymeric substances(EPS) in denitrifying phosphorus removal sludge in the presence of copper ion[J]. Water Research, 2015, 73: 252-264. 